Aufsatz ÖR

Prof. Dr. Stephan Meyer*

\title{
Subjektiver oder objektiver Gefahrenbegriff, "Gefahrenverdacht" und Vorfeldbefugnisse: Dauerbaustellen des Gefahrenabwehrrechts
}

https://doi.org/10.1515/jura-2017-0246

Der Beitrag vermittelt in Teil I die Grundlagen und die dogmatische Bedeutung des Streits um einen subjektiven oder objektiven Gefahrenbegriff. Teil II bespricht, weshalb der sogenannte Gefahrenverdacht dogmatisch nur schwer in den Griff zu bekommen ist. Er behandelt dann die polizeigesetzlichen Eingriffsbefugnisse, die ausdrücklich einen Gefahrenverdacht genügen lassen (»Tatsachen, die eine Annahme rechtfertigen «). Am Schluss steht eine Diskussion der vorherrschenden Auffassung, auch der gewöhnliche Gefahrentatbestand eröffne Eingriffe schon bei Gefahrenverdacht.

\section{Subjektiver vs. objektiver Gefahrenbegriff}

\section{Einführung: Der Gefahrenbegriff im Polizei- und Ordnungsrecht}

\section{a) Definition des Gefahrenbegriffs}

Der Rechtsbegriff »Gefahr" meint einen normativen Schwellenwert. Er definiert das Ausmaß, das eine Schutzgutbedrohung erreichen muss, damit (grundrechts-)eingreifende Polizeimaßnahmen erlaubt sind. Hierin unterscheidet sich der Begriff von der Alltagssprache, die eine "Gefahr« häufig bloß als physische Schädlichkeit versteht (»Die Luftverschmutzung stellt eine Gesundheitsgefahr dar!«). Für letztgenannte Bedeutung steht im Englischen der eigenständige Begriff des »hazard « bereit, der etwa im Unionsrecht vorkommt. In deutschen Sprachfassungen unionsrechtlicher Vorschriften wird "hazard « zuweilen dennoch missverständlich mit »Gefahr« übersetzt. Umge-

*Kontaktperson: Stephan Meyer, der Autor ist apl. Professor für Öffentliches Recht an der Universität Erfurt und Inhaber einer Professur an der Technischen Hochschule Wildau. kehrt fehlt es auch an einer eindeutigen englischen Übersetzung des deutschen Gefahrenbegriffs. Der Rechtsbegriff »risque/risk« bezeichnet den Erwartungsschaden, also das Produkt aus Eintrittswahrscheinlichkeit und Schadenshöhe, ohne damit eine bestimmte normative Schwelle markieren zu wollen.

Die gängige Gefahrendefinition lautet: Eine (konkrete) Gefahr liegt vor, wenn im Falle des ungehinderten Fortgangs eines Geschehensverlaufs mit hinreichender Wahrscheinlichkeit ein Schaden an einem polizeilichen Schutzgut eintreten wird. ${ }^{1}$

Das Abstellen auf Wahrscheinlichkeiten ist erforderlich, weil die Gefahrenabwehr präventiv der Verhinderung eines zukünftigen Ereignisses dient. Die zukünftige Entwicklung ist ihrer Natur nach selbst im Falle einer zutreffenden und vollständigen Kenntnis des gegenwärtigen Sachverhalts immer in gewissem Maße »unsicher«.

\section{b) Die »hinreichende« Wahrscheinlichkeit}

Ab welcher Höhe die Eintrittswahrscheinlichkeit »hinreichend « ist, hängt ab vom Stellenwert des gefährdeten Schutzguts und von der Schwere dessen Schädigung im Falle des Schadenseintritts, kurz: von der zu erwartenden Schadenshöhe. Je größer die Schadenshöhe, desto niedriger darf die Eintrittswahrscheinlichkeit sein, um als »hinreichend « zu gelten (Je-desto-Formel). Auch sehr geringe Wahrscheinlichkeiten können damit im Einzelfall hinreichen.

Da die Gefahrenprognose also lediglich eine Wahrscheinlichkeitsaussage trifft, wird eine positive Prognose (Wahrscheinlichkeit hinreichend) nicht dadurch als »falsch" erwiesen, dass der Schaden ausbleibt. Ebenso wenig wird eine negative Prognose »falsch«, weil der Scha-

1 Siehe die Legaldefinitionen in $\S 2$ Nr. 3 Buchst. a BremPolG, § 3 Abs. 3 Nr. 1 SOG M-V, § 2 Nr. 1 Buchst. a Nds. SOG, § 3 Nr. 3 Buchst. a SOG LSA, § 54 Nr. 3 Buchst. a ThürOBG. 
den eintritt. Angenommen, Eintrittswahrscheinlichkeit und erwartete Schadenshöhe seien sehr gering, so dass nach der Je-desto-Formel keine Gefahr vorliegt. Tritt das Schadensereignis nun doch ein, widerlegt dies alleine nicht die Prognose: Wer gleich beim ersten Wurf eine Sechs würfelt, widerlegt damit schließlich auch nicht, dass die Wahrscheinlichkeit, eine Sechs zu würfeln, lediglich Eins zu Sechs beträgt.

Das Gefahrenabwehrrecht gewährleistet damit eine Lastenverteilung zwischen dem Träger des polizeilichen Schutzguts und dem potentiell Polizeipflichtigen, in dessen Individualrechte die Polizei zur Abwendung des Schadens eingreifen müsste. Unterhalb der Gefahrenschwelle hat der Schutzgutträger die bestehende Möglichkeit des Schadenseintritts als allgemeines Lebensrisiko zu gewärtigen.

\section{c) Die "Störung"}

Ist der Schaden bereits eingetreten und dauert die schädigende Einwirkung fort, spricht man auch von einer "Störung«. Um einen dogmatisch eigenständigen Begriff handelt es sich nicht. Eine gegenwärtige Einwirkung kann in der Gegenwart nicht mehr beseitigt werden, weil die Gegenwart in einer juristischen Sekunde zur Vergangenheit wird. Es geht daher auch bei der Beseitigung von Störungen um Prävention. Die Störung ist Basis einer Gefahrenprognose:

Steht ein Auto im Augenblick im Halteverbot, wird dies auch in der nächsten juristischen Sekunde so sein. Gegen diesen zukünftigen Schaden an einem polizeilichen Schutzgut richtet sich die Gefahrenabwehrmaßnahme.

\section{Auseinandersetzung mit den beiden konkurrierenden Gefahrenbegriffen}

Ob der Sachverhalt die Annahme einer hinreichenden Wahrscheinlichkeit trägt, können in der Entscheidungssituation nur die handelnden Polizei- oder Ordnungsbehörden (im Folgenden kurz: Polizeibehörden) beurteilen.

Der Streit um den objektiven oder subjektiven Gefahrenbegriff gilt einem je unterschiedlichen Umgang mit der Fehleranfälligkeit dieser Beurteilung. Die Entscheidung für einen der beiden Begriffe besitzt Konsequenzen für die Rechtmäßigkeit polizeilichen Handelns und darf daher nicht offen bleiben.

Der objektive Gefahrenbegriff verlangt, dass der Gefahrenprognose stets ein zutreffender Sachverhalt zu Grunde liegen muss - das heißt auch dann, wenn der Sachverhalt erst im Nachhinein vollständig aufzuklären ist. Führen falsche Sachverhaltsannahmen zu einer unzutreffenden Bejahung einer Gefahr, ist dies also auch dann rechtswidrig, wenn den handelnden Beamten der Fehler nicht vorwerfbar ist.

Fall 1: Am Flussufer steht ein Fass mit der Aufschrift »Gift«, aus dem eine Flüssigkeit in den Fluss läuft. Polizeibeamtinnen nehmen einen in der Nähe tätigen Gabelstaplerfahrer als Nichtstörer in Anspruch, um das Fass als Erstmaßnahme vom Flussufer wegzuschaffen und so eine weitere Vergiftung des Flusses zu verhindern. Später stellt sich heraus, dass das Fass mit reinem Wasser gefüllt war.

Nach dem objektiven Gefahrenbegriff ist die Inanspruchnahme des Gabelstaplerfahrers in Fall 1 rechtswidrig, weil der Gefahrentatbestand nicht eröffnet war. Das Einleiten von Wasser in einen Fluss trägt nicht die Gefahrenprognose, der Fluss würde bei Nichteinschreiten mit hinreichender Wahrscheinlichkeit vergiftet werden. Dass die Beamten wegen der Aufschrift davon ausgehen mussten, dass es sich um Gift handelt, spielt für dieses rechtliche Ergebnis keine Rolle.

Der subjektive Gefahrenbegriff geht davon aus, dass es auf eine nachvollziehbare Einschätzung der Lage durch die Beamtinnen vor Ort ankommt. Eine Gefahr besteht demnach, wenn die Polizei aufgrund der erlangten Informationen von der hinreichenden Wahrscheinlichkeit eines Schadens an einem polizeilichen Schutzgut im Falle des ungehinderten Fortgangs ausgeht und diese Annahme vom Standpunkt eines sich in der gleichen Lage befindlichen fähigen, besonnenen und sachkundigen Polizeibeamten (objektivierter Beamter ${ }^{2}$ ) nachvollziehbar ist.

Diese Auffassung hat zur Folge, dass polizeiliches Einschreiten auch dann rechtmäßig ist, wenn tatsächlich keine hinreichende Wahrscheinlichkeit eines Schadenseintritts vorliegt, sofern die gegenteilige Beurteilung durch die handelnden Beamten in der fraglichen Entscheidungssituation nachvollziehbar ist: die sogenannte Anscheinsgefahr (so im Fall 1).

2 Vogel/Tipke/Hoffmann-Riem FS Wacke, 1972, 327 (339). 


\section{Rechtfertigung des subjektiven Gefahrenbegriffs}

\section{a) Bedenken aus objektiver Sicht}

Der objektive Ansatz möchte aus rechtsstaatlichen Gründen vermeiden, dass ein bloß subjektives Meinen des Rechtsanwenders zu einer Tatbestandsvoraussetzung von Eingriffsermächtigungen gerät. ${ }^{3}$ Er anerkennt, dass in polizeilichen Entscheidungssituationen eine vollständige Sachverhaltsaufklärung häufig nicht stattfinden kann und die Rechtsordnung hierauf reagieren muss [unten bei b)]. Der Preis einer subjektiven Relativierung des materiellen Rechts erscheint ihm indes zu hoch. Der Schutz der Polizeibeamten vor persönlichen Folgen unvermeidbarer Fehleinschätzungen erfordere keinen subjektiven Gefahrenbegriff [bei c)]. Des Weiteren zeige der verwaltungsprozessuale Eilrechtsschutz, dass Zeitdruck nicht zwingend eine Subjektivierung gebietet [bei d)]. Und schließlich ziehe der subjektive Gefahrenbegriff Folgeprobleme nach sich, die die polizeiliche Verantwortlichkeit betreffen [bei I.5.].

\section{b) Entscheidungsdruck als Motiv für den subjektiven Gefahrenbegriff}

Auch der objektive Gefahrenbegriff trägt dem Umstand Rechnung, dass die zukünftige Entwicklung stets ungewiss ist, denn er lässt ebenso wie der subjektive Ansatz ein Wahrscheinlichkeitsurteil als Gefahrentatbestand genügen. ${ }^{4}$ Aufgrund der Je-desto-Formel darf die unterstellte Eintrittswahrscheinlichkeit einzelfallabhängig sogar äußerst gering sein. Von diesem funktionalen Ausgangspunkt erscheint dann aber folgerichtig, wenn der Gefahrenbegriff nicht nur die generelle Ungewissheit zukünftiger Entwicklungen als eine relevante Quelle von Unsicherheit anerkennt, sondern zudem eine unvermeidbar unvollständige Sachverhaltskenntnis. Ein »Mehr» an Kenntnis kann - in der eiligen Entscheidungssituation - ebenso objektiv unmöglich sein ${ }^{5}$ wie eine Zukunftsprognose mit einer Eintrittswahrscheinlichkeit von Eins. Zwar weiß man es hinterher oft besser und trägt das Verwaltungsrecht dem im

3 Geis/Götz Allgemeines Polizei- und Ordnungsrecht, 16. Aufl. 2017, $\S 6$ Rn. 18; Kingreen/Poscher Polizei- und Ordnungsrecht, 9. Aufl. 2016, § 4 Rn. 36 ff.; Poscher Gefahrenabwehr, 1999, 98, 105 f.

4 Dazu oben bei I.1.

5 Ähnlich Schoch/ders. BesVwR, 15. Aufl. 2013, 2. Kap. Rn. 141; ders. JURA 2003, 472 (473). Der subjektive Gefahrenbegriff stellt auf einen objektivierten Polizeibeamten ab, dazu oben bei I.2.
Allgemeinen auch Rechnung. ${ }^{6}$ Während in sonstigen Regelungsbereichen jedoch zu erwarten ist, dass die Behörde den Sachverhalt gemäß § 24 VwVfG vor der Sachbescheidung vollständig aufklären kann und Fehlentscheidungen eine pathologische Ausnahme bleiben, ist für die Gefahrenabwehrbehörden eine vollständige Aufklärung regelmäßig ausgeschlossen, sofern der gesetzliche Auftrag der effektiven Gefahrenabwehr erfüllt werden soll. ${ }^{7}$ In der Folge geriete die Rechtsordnung widersprüchlich: Polizeibehörden würde eine Aufgabenerledigung gesetzlich abverlangt, deren Rechtmäßigkeitsbedingungen in der Praxis allenfalls erratisch zu erfüllen wären. Die Rechtmäßigkeit staatlichen Handelns hinge also nicht mehr von der bewussten Orientierung des Staatsorgans am Recht ab, sondern von empirischen Umständen, die zum gesetzlich geforderten Entscheidungszeitpunkt nicht erkennbar sind.

Dem Staat sollte rechtmäßiges Verhalten jedoch als gezielte Orientierung am Recht möglich sein, und nicht nur zufällig. Gerade hierin liegt die Funktion des »Rechtscodes« - also die Unterscheidung rechtmäßig/rechtswidrig -, der staatliches Handeln für den Bürger vorhersehbar macht, indem er den Staatsorganen ihre Handlungsorientierung bereitstellt. ${ }^{8}$ Dies spricht für den subjektiven Gefahrenbegriff.

Weil Zweck des Gefahrentatbestands die Unsicherheitsverarbeitung ist, erscheint zudem plausibel, dass die Richtigkeit des angenommenen Sachverhalts Bestandteil der Gefahrenprognose ist (d.h. die Richtigkeit muss bloß hinreichend wahrscheinlich sein), nicht etwa nur deren Grundlage (Richtigkeit müsste gewiss erscheinen). ${ }^{9}$

6 Siehe insb. § 51 Abs. 1 Nr. 2 VwVfG: Wiederaufgreifen aufgrund neuer Beweismittel.

7 A. A. hins. eines Unterschiedes Kingreen/Poscher (Fn. 3), § 4 Rn. 44; Poscher (Fn. 3), 101.

8 In diesem Sinne auch Möstl Die staatliche Garantie für die öffentliche Sicherheit und Ordnung, 2002, 169, 172, 174; Freund GA 1991, 387 (391 ff.).

9 Für die erste Variante (Bestandteil): Bäcker (Fn. 14), 82f.; Bonin Grundrechtsschutz durch verfahrensrechtliche Kompensation bei Maßnahmen der polizeilichen Informationsvorsorge, 2012, 204; Vogel/Tipke/Hoffmann-Riem (Fn. 2), 336; Möstl (Fn. 8), 169ff.; Schoch/ ders. (Fn. 5), Rn. 142; ders. JURA 2003, 472 (474). Differenzierend Ehlers DVBl 2003, 336 (338). Für die zweite Variante (Grundlage): BVerwGE 116, 347 (352); Geis/Götz (Fn. 3), § 6 Rn. 31; Kral Die polizeilichen Vorfeldbefugnisse als Herausforderung für Dogmatik und Gesetzgebung des Polizeirechts, 2012, $36 \mathrm{ff}$. Zur Diskussion Albers Die Determination polizeilicher Tätigkeit in den Bereichen der Straftatenverhütung und der Verfolgungsvorsorge, 2001, $52 \mathrm{ff}$. 


\section{c) Die persönliche Verantwortlichkeit der handelnden Amtswalter}

Befürworter des objektiven Gefahrenbegriffs machen zu Recht geltend, ${ }^{10}$ dass die nachträgliche Bewertung des polizeibehördlichen Handels als rechtswidrig mit einer Freistellung der Beamten vom Vorwurf eines Dienstvergehens vereinbar ist, soweit ein Tatbestandsirrtum nicht schuldhaft auftrat. ${ }^{11}$ Der Schutz der Polizeibeamtinnen vor nachteiligen persönlichen Folgen des Entscheidens unter Zeitdruck ist alleine also kein Argument für den subjektiven Gefahrenbegriff. Jedoch kann die Rechtsordnung sich nicht nur um die Bewertung des persönlichen Handelns der Beamten sorgen, sondern sie muss ebenso und vor allem den Staat selbst als Rechtsträger im Blick haben, dem das Handeln seiner Polizeibehörden bzw. -beamten zugerechnet wird. ${ }^{12}$ Auch für den Staat als Rechtssubjekt hat Ultra posse nemo obligatur zu gelten. Hält man dagegen die "rechtliche Bewertung der Gefahrenabwehrmaßnahme in erster Linie [nur] für die Entscheidung der Rechtsbehelfsinstanzen über die Aufhebung der Maßnahme [...] maßgeblich ${ }^{13}{ }^{13}$ so verengt dies die Funktion des Gesetzesrechts zu sehr auf die Ex-post-Kontrollperspektive. ${ }^{14}$ Es ist die Kernidee der Unterscheidung von Verhalten als rechtmäßig/rechtswidrig, dass Rechtsbrüche - zumal solche des Staates - möglichst gar nicht erst auftreten sollen.

\section{d) Vergleich mit dem Eilrechtsschutz}

Im Eilrechtsschutz ist die Sach- und Rechtslage trotz der Eilbedürftigkeit eingehend zu prüfen. ${ }^{15}$ In Fällen indes, in denen der Zeitdruck solche Sorgfalt ausschließt, ist nach überwiegender Meinung ${ }^{16}$ Prüfungsmaßstab nicht die -

$10 \operatorname{Poscher}$ (Fn. 3), $98 \mathrm{ff}$.

$11 \S 77$ Abs. 1 Satz 1 BBG und $\S 47$ Abs. 1 Satz 1 BeamtStG definieren das Dienstvergehen als schuldhafte Pflichtverletzung. Der unvermeidbare Tatbestandsirrtum lässt nur die Schuld, nicht aber die Verletzung der Dienstpflicht zur Einhaltung des Polizeirechts entfallen. Dienstlich »erlaubt« ist daher ein Handeln der Beamten bei sorgfältiger aber irriger Bejahung einer Gefahr nicht (a. A. Poscher (Fn. 3), 99f., auch 102, 128, 147 ff.).

12 So auch Möstl (Fn. 8), $172 \mathrm{ff}$.

13 Poscher (Fn. 3), 110.

14 Vgl. Schmidt-Aßmann/Hoffmann-Riem/ders. Verwaltungsorganisationsrecht als Steuerungsressource, 1997, 355 (388). Siehe auch Bäcker Kriminalpräventionsrecht, 2015, $89 \mathrm{f}$.

15 Dazu Kingreen/Poscher (Fn. 3), § 4 Rn. 44.

16 BVerfGE 110, 77 (87 f.); BVerfG EuGRZ 2016, 698 (699f.); DVBl 2013, 367 (368, Abs. 18); stRspr. Ausf. Quaas/Zuck/Funke-Kaiser Prozesse in Verwaltungssachen, 2. Aufl. 2011, § 4 Rn. 252ff.; Kopp/Schenke/ders. zeitnah nicht zu klärende - materielle Rechtslage, sondern jedenfalls im Aussetzungsverfahren eine Interessenabwägung. Vergleichbares wird für die einstweilige Anordnung angenommen (Interessen-/Folgenabwägung bei offener Erfolgsaussicht in der Hauptsache, str.). ${ }^{17}$ Auch beim Eilrechtsschutz reagiert die Rechtsordnung auf den Zeitdruck also mit materiell-rechtlichen ${ }^{18}$ Lockerungen. Erweist sich die Anordnung als ungerechtfertigt, trifft zudem nicht den Staat die Schadensersatzpflicht, sondern den Antragsteller, § 123 Abs. 3 VwGO i. V.m. § 945 ZPO. Auch damit markiert die Rechtsordnung, dass der Staat das Risiko einer Fehlentscheidung nicht selbst tragen soll, sondern ein Veranlasser. ${ }^{19}$ Im Übrigen immunisiert das auch für den Eilrechtsschutz geltende Spruchrichterprivileg aus §839 Abs. 2 Satz 1 BGB die Rechtskraft der Anordnung gegen die Geltendmachung von Haftungsansprüchen aus einer Fehlentscheidung. ${ }^{20}$ Im Ergebnis zeigt sich: Der Staat schirmt sich im Falle des Eilrechtsschutzes sogar noch stärker ${ }^{21}$ gegen Rechtsfolgen von Bewertungsfehlern bei einer Entscheidung unter Unsicherheit ab als im Falle des subjektiven Gefahrenbegriffs.

\section{Subjektiver Gefahrenbegriff und polizeirechtliche Verantwortlichkeit}

\section{a) Der Verantwortliche bei einer Anscheinsgefahr}

Nach dem objektiven Gefahrenbegriff fehlt es bei einer "Anscheinsgefahr" an einer polizeirechtlichen Gefahr und kann es daher auch keinen Störer geben. Nach dem herrschenden subjektiven Begriff handelt es sich hingegen um

VwGO, 22. Aufl. 2016, § 80 Rn. 152ff., bes. Rn. 158. Kritik vor allem bei Schneider/Bier/Schoch/ders. VwGO, § 80 Rn. 369 ff. (Stand: EL 22/ 2011), § 123 Rn. 65 f. (Stand: EL 26/2014).

17 Diskussion bei Schneider/Bier/Schoch/ders. VwGO, § 123 Rn. 64a ff. (Stand: EL 26/2014); zustimmend Hufen Verwaltungsprozessrecht, 10. Aufl. 2016, § 33 Rn. 16; Sodan/Ziekow/Puttler VwGO, 4. Aufl. 2014, § 123 Rn. 100 f.

18 Poscher (Fn. 3), 165f. mit Fußm. 113 sieht im »Glaubhaftmachen« gem. § 123 Abs. 3 VwGO i.V.m. § 920 ZPO zu Recht eine verfahrensrechtliche Lockerung. Sofern jedoch der Prüfungsmaßstab gegenüber dem des Hauptsacheverfahrens vollständig ausgetauscht wird (Interessen-/Folgenabwägung statt die den Fall mutmaßlich bestimmende materielle Rechtslage), handelt es sich m.E. um eine materiell-rechtliche Lockerung.

$19 \mathrm{Vgl}$. Sodan/Ziekow/Puttler (Fn. 17), § 123 Rn. $143 \mathrm{ff}$.

20 W.-R. Schenke JZ 2005, 680 (683f.), bezugnehmend auf BGHZ 161, 298.

$21 \mathrm{Zu}$ Erleichterungen für den Anscheinsstörer auf der Sekundärebene bei I.5. 
eine tatbestandsmäßige Gefahr, ${ }^{22}$ daher ergeben sich auf den ersten Blick für die Verantwortlichkeit keine dogmatischen Besonderheiten. Störer ist der, der die Anscheinsgefahr verursacht hat.

Beispiel: Die Polizei beobachtet eine Person, die nachts die Umzäunung eines Privatgeländes überwindet. Es handelt sich bei der Person jedoch um einen Berechtigten. Die Person ist der Verursacher der Schutzgutbedrohung, von der die Beamten nachvollziehbar ausgehen, und somit Verhaltensstörer (zumeist "Anscheinsstörer" genannt).

\section{b) Der nur scheinbar Verantwortliche}

Von der Verantwortlichkeit für eine Anscheinsgefahr analytisch zu unterscheiden ist eine nur scheinbare Verantwortlichkeit für eine (Anscheins-) ${ }^{23} \mathrm{Gefahr}$.

Fall 2: Die Polizei bemerkt einen Ölfilm auf einem See und ein Ölfass am Ufer. Zur Gefahrenabwehr nimmt sie daher diejenige Person in Anspruch, die das Fass augenscheinlich dort abgeladen hat. In Wahrheit stammt der Ölfilm jedoch nicht von dem Fass, sondern von einem verölten Gegenstand, den ein Unbekannter in den See geworfen hat.

Die herrschende Meinung subsumiert auch derartige Fälle der Verhaltens- oder Zustandsverantwortlichkeit. ${ }^{24}$

\section{c) Mindermeinung zur Verantwortlichkeit}

Nach einer beachtlichen Mindermeinung kommt es bei a) und b) darauf an, ob der Person vorwerfbar ist, dass ihr eigenes Verhalten sie als Verursacher der (Anscheins-)Gefahr erscheinen lässt. Ist dies nicht der Fall, soll es sich nicht um einen Störer handeln und allenfalls eine Inanspruchnahme als Nichtstörer in Betracht kommen. ${ }^{25}$

22 Aus jüngerer Zeit BVerwGE 153, 211 (214, Rn. 14) sowie VGH München BayVBl 2017, 303 (303, Abs. 17); BayVBl 2016, 341 (345, Abs. 49); OVG Koblenz, Urt. v. 27. 3. 2014 - 7 A 10993/13, juris Rn. 29; OLG Brandenburg a.d.H., Beschl. v. 8. 5. 2014 - 11 Wx 8/11, juris. Grundlegend: BVerwGE 45, 51 (60).

23 Für den subjektiven Gefahrenbegriff ist die Anscheinsgefahr eine tatbestandsmäßige Gefahr, so dass sie mit erfasst wird.

24 Selmer/v. Münch/Breuer, GS Martens, 1987, 317 (335ff.); Thiel Polizei- und Ordnungsrecht, 3. Aufl. 2016, § 8 Rn. 99; VGH Mannheim NVwZ-RR 1991, 24; OVG Münster NJW 1993, 2698.

25 Etwa Lisken/Denninger/Rachor Hdb Polizeirecht, 5. Aufl. 2012, M Rn. 49 ff.; Schenke Polizei- und Ordnungsrecht, 9. Aufl. 2016, § 4 Rn. $255 \mathrm{ff}$.
Nach der herrschenden Meinung ist die Inanspruchnahme der Person als Störer in Fall 2 rechtmäßig, nach der Mindermeinung handelt es sich um einen Nichtstörer (sofern das Abladen des Fasses als solches rechtmäßig ist).

Folgt man der Mindermeinung, ist zu gewärtigen, dass nicht ohne weiteres auf die Inanspruchnahme von Notstandspflichtigen als Ermächtigungsgrundlage abgestellt werden kann, sollte sich der Eingriffsadressat im Nachhinein als Nichtstörer erweisen. Hierfür fordern die einschlägigen Vorschriften eine qualifizierte Gefahr. ${ }^{26}$

\section{Subjektiver Gefahrenbegriff und Sekundärebene des polizeilichen Handelns}

\section{a) Die herrschende Auffassung}

$\mathrm{Zu}$ Problemen führt der subjektive Gefahrenbegriff bei der Frage, ob der Polizeipflichtige in Fällen der Anscheinsgefahr gegenüber dem Rechtsträger der Polizei Kostenersatz zu leisten hat, bzw., umgekehrt, ob er im Falle erlittener Schäden einen Entschädigungsanspruch besitzt.

Die Geltendmachung derartiger Ansprüche erfolgt nämlich zu einem Zeitpunkt, zu dem der Sachverhalt in der Regel vollständig aufgeklärt ist. Es ist daher bekannt, ob tatsächlich eine hinreichende Wahrscheinlichkeit eines Schadenseintritts bestand. Eine Kostenersatzpflicht bzw. ein fehlender Entschädigungsanspruch können daher unbillig erscheinen.

Fall 3: Mit schwankendem Gang nähert sich ein Taxifahrer seinem Fahrzeug, steigt ein und will losfahren. Die Polizei hält ihn davon ab. Der Taxifahrer macht eine Beinverletzung geltend. Die Beamten unterbinden die Weiterfahrt. Die Angabe des Taxifahrers stellt sich später als wahrheitsgemäß heraus. Da jedoch eine (Anscheins-)Gefahr vorlag, ist der Taxifahrer Störer. Für den erlittenen Verdienstausfall stünde ihm daher an sich keine Entschädigung zu. ${ }^{27}$

Fall 4: Eine Gruppe Fußballfans zieht randalierend durch die Stadt. Ein Anhänger (A) des betreffenden Vereins, der von der Randale nichts bekommen hat, tritt gerade aus einem Lokal heraus, als die Gruppe an diesem vorbeikommt. A hält die Gruppe für harmlose Fußballbegeisterte und schließt sich ihr an. Kurz darauf wird die ganze Gruppe von der Polizei in Gewahrsam genommen, um weitere Ausschreitungen zu verhindern. Aufgrund der Aufmachung des A und seines Mitmarschierens durfte die Polizei nachvollziehbar davon ausgehen, dass auch A in Kürze an Gewalttätigkeiten beteiligt sein würde. A weist zwar

26 Dazu Schoch JURA 2007, 676 (678f.).

27 Siehe die Vorschriften in Fn. 37. 
darauf hin, dass er Unbeteiligter sei. Die Polizei hält dies aber nachvollziehbar für eine Schutzbehauptung. Zeugenaussagen bestätigen später, dass A unbeteiligt war und nicht wissen konnte, dass es sich bei der Gruppe um Randalierer handelte. Da A nach herrschender Meinung Störer ist, könnte die Polizeibehörde an sich einen Gebührenbescheid gegen A zur Heranziehung zu den Kosten der Ingewahrsamnahme erlassen. ${ }^{28}$

Die herrschende Meinung korrigiert diese Rechtsfolgen, indem sie der Entscheidung über die Kostentragung bzw. über eine Entschädigung den aktuellen Kenntnisstand zugrundelegt (sog. »Ex-post-Perspektive«). Im Falle einer Anscheinsgefahr liegt zwar eine Gefahr vor, die rechtmäßiges Einschreiten ermöglicht (»Primärebene«). Weil sich im Nachhinein erweist, dass ein Schadenseintritt nicht mit hinreichender Wahrscheinlichkeit zu erwarten war, ist der Störer indes nicht kostentragungspflichtig (Fall 4) und kann vielmehr Entschädigung für ihm entstandene Schäden geltend machen (Fall 3). Er wird insoweit dem Nichtstörer in entsprechender Anwendung der für diesen geltenden Entschädigungsvorschriften gleichgestellt.. ${ }^{29}$ Ebenso verfahren wird bei dem primär als Störer in Anspruch genommenen, nur scheinbaren Verantwortlichen einer (Anscheins-)Gefahr ${ }^{30}$ (auf der Sekundärebene dann gelegentlich »anscheinsbetroffener Nichtstörer « genannt).

Das gilt nur dann nicht, wenn der Störer die Anscheinsgefahr oder den Anschein, der Verantwortliche für eine Gefahr zu sein, in vorwerfbarer Weise und damit $z u$ rechenbar verursacht hat. ${ }^{31}$ Das ist dann der Fall, wenn er hätte erkennen können, dass sein Verhalten als gefahrenverursachend eingeschätzt werden würde und ihm entweder das Unterlassen dieses Verhaltens oder zumindest eine Vorkehrung gegen Missverständnisse zumutbar war.

Fall 5: Morgens klingelt eine Stunde lang der Wecker in einer Wohnung, in der eine gebrechliche Dame wohnt. Nachbarn sorgen sich, dass dieser etwas zugestoßen sein könnte und alarmie-

28 In Anlehnung an VGH Mannheim ESVGH 61, 198 = DVBl 2011, 626. Das Gebührenverzeichnis Innenministerium BW umfasst den Unterbindungsgewahrsam als Gebührentatbestand (Nr. 15.2). Vgl. Kugelmann/Alberts JURA 2013, 898 (905).

29 BGHZ 117, 303 (307f.). Entsprechend BGHZ 126, 279 (283f.); BGH NJW 1996, 3151 (3152). Zust. Lisken/Denninger/Rachor (Fn. 25), Rn. 42ff.; Gusy Polizei- und Ordnungsrecht, 10. Aufl. 2017, § 8 Rn. 460; Kugelmann/Alberts JURA 2013, 898 (900f.); Schoch/ders. (Fn. 5), Rn. 421. Zum Sekundärrechtsschutz des Nichtstörers Schoch JURA 2007, 676 (682ff.).

$30 \mathrm{Zu}$ diesem bei I.4.b) und c).

31 BGHZ 117, 303 (308). Aus jüngerer Zeit VGH München BayVBl 2017, 303 (304f., Abs. 23 ff.); VGH Mannheim VBlBW 2014, 56 (58) sowie ESVGH 61, 198 = DVBl 2011, 626; VG München, Urt. v. 23. 11. 2016 - M 7 K 15.3762, juris; VG Saarlouis, Urt. v. 22. 9. 2016 - 6 K 493/15, juris. ren die Polizei. Da niemand öffnet, lässt die Polizei die Tür von einem Schlüsselnotdienst öffnen. Später stellt sich heraus, dass die Dame verreist ist und vergessen hatte, den Timer abzustellen. Dadurch hat sie die Anscheinsgefahr vorwerfbar verursacht und die Polizei kann sie zu den Kosten der Türöffnung heranziehen. ${ }^{32}$

\section{b) Kritische Stimmen}

Die unterschiedliche Behandlung der Primär- und der Sekundärebene, die als Folge des subjektiven Gefahrenbegriffs auftritt, stößt auf Kritik. Der Differenzierung fehle eine rechtliche Grundlage. ${ }^{33}$

Richtig ist, dass das Polizei- bzw. Kostenrecht zumindes ${ }^{34}$ den Ersatz besonderer Kosten (insb. der unmittelbaren Ausführung bzw. Ersatzvornahme, ${ }^{35}$ und der Sicherstellung/Verwahrung) von einem Störer fordert. ${ }^{36}$ Umgekehrt behält das Polizeirecht eine Entschädigung dem Nichtstörer vor. ${ }^{37}$ Anhaltspunkte für Ausnahmen liefert der Wortlaut nicht.

Der herrschenden Meinung ist indes zuzubilligen, dass sie - methodisch nicht von vornherein unzulässig einen teleologischen Auslegungsschwerpunkt einnimmt und aus Gründen der Handlungsfähigkeit der Polizei eine abweichende Bewertung der Verantwortlichkeit auf der Primärebene und der Sekundärebene in das geltende Polizei-/Kostenrecht hineinliest, welche jedenfalls nicht im offenen Widerspruch ${ }^{38}$ zum Wortlaut steht. Als verfassungskonforme Auslegung ${ }^{39}$ des Kostentragungs- und Entschädigungsrechts erscheint dies noch vertretbar.

32 In Anlehnung an VGH Mannheim VBlBW 2011, 153. Je nach Landesrecht zu ersetzen sind die Kosten für die unmittelbare Ausführung oder für die Ersatzvornahme als Sofortvollzug. Dazu Möstl/Trurnit/ Kastner BeckOK PolR BW, 6. Ed., 2017, § 8 PolG Rn. $1 \mathrm{ff}$.

33 Kingreen/Poscher (Fn. 3), §4 Rn. 67 ff.; Schenke (Fn. 25), §4 Rn. $253 \mathrm{ff}$.

34 Hinsichtlich sonstiger Kosten bestehen landesrechtliche Unterschiede. Näheres regeln die jeweiligen Gebührenordnungen für Amtshandlungen der Polizei (Bsp. in Fußn. 28).

35 Abhängig vom Landesrecht.

36 Im einzelnen Kugelmann/Alberts JURA 2013, $898 \mathrm{ff}$.

37 Für die Polizei: § 55 Abs. 1 PolG BW, Art. 70 Abs. 1 PAG (Bayern), $\S 59$ Abs. 1 Nr. 1 ASOG, $\S 70$ BbgPolG i. V.m. § 38 Abs. 1 Buchst. a OBG (Bbg), § 56 Abs. 1 BremPolG, § 10 Abs. 3 Satz 1 SOG (Hambg.), § 64 Abs. 1 Satz 1 HSOG, $\S 72$ Abs. 1 SOG M-V, § 80 Abs. 1 Satz 1 Nds. SOG, $\S 67$ PolG NRW i.V.m. § 39 Abs. 1 Buchst. a OBG (NRW), § 68 Abs. 1 Satz 1 POG (Rh.-Pf.), § 68 Abs. 1 Satz 1 SPolG (Saarl.), § 52 Abs. 1 SächsPolG, § 69 Abs. 1 Satz 1 SOG LSA, § 221 Abs. 1 LVwG (S.-H.), § 68 Abs. 1 Satz 1 PAG (Thür.), § 51 Abs. 1 Nr. 1 BPolG.

38 So auch BGHZ 117, 303 (307).

39 Die Heranziehung zu Kosten für eine polizeiliche Maßnahme, zu der eine Person selbst keinerlei Veranlassung gegeben hat, erschiene willkürlich. Erleidet eine Person einen Schaden aufgrund einer poli- 


\section{Der Gefahrenverdacht}

\section{Definition}

Ein Gefahrenverdacht liegt vor, wenn die Polizei das Vorliegen einer hinreichenden Wahrscheinlichkeit eines Schadenseintritts lediglich für möglich hält. Es besteht ein Informationsdefizit, das der Polizei bewusst ist, und das die Bejahung der hinreichenden Wahrscheinlichkeit (noch) nicht zulässt. Die normative Schwelle für eingreifende Gefahrenabwehrmaßnahmen ist also noch nicht überschritten. ${ }^{40}$

\section{Der Gefahrenverdacht als dogmatische Herausforderung}

\section{a) Einführung: Weitere Beurteilungsfehler jenseits der Anscheinsgefahr}

Um sich dem dogmatischen Problem zu nähern, werden zuerst zwei Sonderfälle besprochen, die noch keinen Gefahrenverdacht enthalten.

Klar ist, dass nach dem subjektiven Gefahrenbegriff eine Gefahr vorliegt, wenn die Beamten die hinreichende Wahrscheinlichkeit eines bevorstehenden Schadenseintritts pflichtgemäß aber irrig bejahen (Anscheinsgefahr). Wie verhält es sich jedoch, wenn die Beamten die hinreichende Wahrscheinlichkeit pflichtgemäß abschließend verneinen, diese jedoch in der fraglichen Lage tatsächlich vorlag?

Fall 6: Polizeibeamte haben den Eindruck, dass die Bremsleuchten des vorausfahrenden Fahrzeugs defekt sein könnten. Sie sind sich jedoch nicht sicher, da aufgrund starken Sonnenlichteinfalls die Helligkeit der Bremsleuchten überdeckt sein könnte. Nachdem das Fahrzeug innerhalb eines Schattenwurfs zum Stillstand gekommen ist, ist dann aber klar zu erkennen, dass die Bremsleuchten funktionieren. Ohne weitere Maßnahmen setzen die Beamten ihre Fahrt fort. Tatsächlich liegt jedoch ein Wackelkontakt vor; als das Fahrzeug wieder in Bewegung ist, fallen die Leuchten erneut aus und es geschieht alsbald ein Auffahrunfall.

zeilichen Maßnahme, ohne selbst eine zurechenbare Ursache gesetzt zu haben, erbringt sie ein Sonderopfer (dazu Kießling JURA 2016, 483 (484ff.); Kugelmann/Alberts JURA 2013, 898 (900); Schoch JURA 2007, 676 (682)), das verfassungsrechtlich ausgleichspflichtig ist.

40 BVerwGE 116, 347 (351f.); OVG Münster, Beschl. v. 6. 8. 2015 - 5 B 908/15, juris Rn. 5; NVwZ-RR 2012, 470 (471); OVG Bautzen, SächsVBl 2014, 240 (241, Abs. 6); VGH Mannheim VBlBW 2014, 56 (57); VG Münster, Urt. v. 1. 3. 2017 - 5 K 1276/16, juris Rn. 32; VG Berlin, Urt. v. 25. 8. 2016 - $1 \mathrm{~K}$ 318.14, juris Rn. 19. Entsprechend Schoch/ders. (Fn. 5), Rn. 146; Gusy (Fn. 29), § 3 Rn. 113, § 4 Rn. 197.
Lag trotz der pflichtgemäßen Annahme der Beamten, die Leuchten seien in Ordnung, polizeirechtlich eine Gefahr vor?

Fall 7: Wie Fall 6, aber: Hätten die Beamten dem Fahrer entgegen ihrer pflichtgemäß gewonnenen Überzeugung von der Funktionstüchtigkeit der Bremsleuchten die Weiterfahrt untersagt also insoweit jedenfalls aus ihrer subjektiven Sicht pflichtwidrig gehandelt -, wäre dann dieser Eingriff wegen des Handelns entgegen der eigenen pflichtgemäßen Lagebeurteilung polizeirechtswidrig oder wäre er - da eine hinreichende Wahrscheinlichkeit einer Schutzgutbeeinträchtigung tatsächlich bestand trotzdem rechtmäßig?

Und der zweite Sonderfall: Wie verhält es sich, wenn die Beamten insofern pflichtwidrig eine Gefahr bejahen, als ein umsichtiger Beamter in der gleichen Situation nicht von einer Gefahr ausgegangen wäre, die hinreichende Wahrscheinlichkeit einer bevorstehenden Schutzgutbeeinträchtigung aber tatsächlich vorlag?

Fall 8: In einem Freilufttheater, das als ein solches ganz klar erkennbar ist, findet eine Aufführung statt. Im Rahmen einer Spielszene bedroht ein Protagonist einen anderen Darsteller mit einer Schusswaffe. Ein vorbeikommender Polizist überreagiert und überwältigt den Darsteller. Später stellt sich heraus, dass die Theaterwaffe von einem Neider gegen eine Gaspistole ausgetauscht wurde, damit ein Schauspielkollege während des Stücks verletzt werde.

Ist im Rahmen des subjektiven Gefahrenbegriffs die pflichtgemäße Beurteilung immer allein maßgeblich für die Eröffnung des Gefahrentatbestands, dann lag in den Fällen 7 und 8 keine Gefahr vor und das Einschreiten war jeweils rechtswidrig.

\section{b) Die überwiegende Meinung zu diesen Beurteilungsfehlern}

Davon geht die überwiegende Meinung indes nicht aus. ${ }^{41}$ Vielmehr soll dann, wenn die hinreichende Wahrscheinlichkeit eines bevorstehenden Schadenseintritts tatsächlich besteht, der Gefahrentatbestand in jedem Falle eröffnet sein. $\mathrm{Zu}$ diesem Ergebnis kann man nur gelangen, wenn man den subjektiven Gefahrenbegriff modifiziert. Er müsste um den objektiven Gefahrenbegriff als ein weiteres - sowohl alternatives als auch additives - Tatbestandselement angereichert werden. Für die Eröffnung des Gefahrentatbestands reicht dann, wenn entweder nur der subjektive Gefahrenbegriff (= Anscheinsgefahr) oder

41 Von Schenke (Fn. 25), § 3 Rn. 79a, als »wohl [.] die hM« bezeichnet; auch Paeffgen GA 2014, 638 (651). 
nur der objektive Gefahrenbegriff (hinreichende Schadenswahrscheinlichkeit, die pflichtwidrig oder anderweitig nicht erkannt wird) erfüllt ist oder beide Gefahrenbegriffe erfüllt sind (anzustrebender Regelfall; Die pflichtgemäße Bejahung einer hinreichenden Schadenswahrscheinlichkeit entspricht den tatsächlichen Gegebenheiten). Geht man von einem solchen modifizierten subjektiven Gefahrenbegriff aus, dann lag in den Fällen 7 und 8 eine Gefahr vor.

\section{c) Für diesen modifizierten subjektiven Gefahrenbegriff ${ }^{42}$ sind nun für den Gefahrenverdacht vier Fallunterscheidungen zu treffen.}

(1) Nichteinschreiten/hinreichende Schadenswahrscheinlichkeit besteht nicht

Bei Gefahrenverdacht gehen die Beamten pflichtgemäß davon aus, dass sie die hinreichende Wahrscheinlichkeit des Schadenseintritts noch nicht bejahen können. Entspricht diese Bewertung den tatsächlichen Verhältnissen, ist das Nichteinschreiten evident rechtmäßig.

(2) Nichteinschreiten/hinreichende Schadenswahrscheinlichkeit liegt vor

Schreiten die Beamten wegen der pflichtgemäßen Verneinung einer hinreichenden Schadenswahrscheinlichkeit nicht ein, liegt in Wahrheit aber eine hinreichende Schadenswahrscheinlichkeit vor, dann führt das objektive Element des modifizierten subjektiven Gefahrenbegriffs dazu, dass eine Gefahr polizeirechtlich zu bejahen ist. Das Nichteinschreiten erscheint jedenfalls dann materiell polizeirechtswidrig, wenn das Entschließungsermessen auf Null reduziert war.

Diese Konstellation macht deutlich, dass die bei $b$ ) referierte überwiegende Meinung, wonach im Falle einer tatsächlich bestehenden hinreichenden Schadenswahrscheinlichkeit der Gefahrentatbestand immer eröffnet sein soll (unabhängig von der pflichtgemäßen Einschätzung der Beamten), mit dem Zweck des subjektiven Gefahrenbegriffs schwer vereinbar ist. Der subjektive Gefahrenbegriff soll verhindern, dass die Rechtmäßigkeit staatlichen Handelns zu einem bloßen Zufallsprodukt gerät. Genau ein derartiges Risiko wird hiermit aber an anderer Stelle in die Gefahrenabwehrrechtsdogmatik wieder eingeführt. Dies gilt umso mehr, als die Fallgruppe 2 nicht nur bei Gefahrenverdacht auftreten kann, sondern auch dann, wenn die Beamten pflichtgemäß einen Schadenseintritt praktisch ausschließen (Fall 6). Näher läge, auch für die pflichtgemäße Verneinung einer hinreichenden Schadenswahrscheinlichkeit von einem ausschließlich subjektiven Gefahrenbegriff auszugehen, das heißt die Eröffnung des Gefahrentatbestands dann zu verneinen.

(3) Einschreiten/hinreichende Schadenswahrscheinlichkeit besteht nicht

Schreiten die Beamten entgegen ihrer pflichtgemäßen Verneinung einer hinreichenden Schadenswahrscheinlichkeit - das heißt nur aufgrund der erkannten Möglichkeit eines Schadens - ein und besteht eine hinreichende Wahrscheinlichkeit tatsächlich nicht, dann ist der Gefahrentatbestand sowohl nach dem subjektiven als auch dem objektiven Element des modifizierten subjektiven Gefahrenbegriffs nicht eröffnet und das Einschreiten rechtswidrig.

(4) Einschreiten/hinreichende Schadenswahrscheinlichkeit liegt vor

Diese Lage entspricht weitgehend dem Fall 8. Dort hätte eine pflichtgemäße Sachverhaltsbeurteilung zur Verneinung einer Gefahr geführt, obwohl eine hinreichende Schadenswahrscheinlichkeit tatsächlich vorlag. Die vorwerfbare Fehleinschätzung der Situation (Verkennung der Theateraufführung als Bedrohungslage) führte nur zufällig zum Ergebnis, dass das Eingreifen des Beamten einen tatsächlich drohenden Schaden abgewendet hat. Davon unterscheidet sich die vorliegende Fallgruppe nur dadurch, dass der Beamte den Sachverhalt hier pflichtgemäß beurteilt hat (er weiß, dass sein Informationsstand die Bejahung einer hinreichenden Schadenswahrscheinlichkeit noch nicht trägt), er sich über diese Einsicht aber aufgrund der bloßen Möglichkeit eines Schadens hinwegsetzt und am Ende dann »Glück hat", dass eine hinreichende Schadenswahrscheinlichkeit tatsächlich bestand. Wie im Fall 8 ist der Gefahrentatbestand eröffnet, weil jedenfalls das objektive Element des modifizierten subjektiven Gefahrenbegriffs erfüllt ist. Gegen dieses rechtliche Ergebnis sind die gleichen Bedenken zu erheben wie bei Fallgruppe 2 und wäre auch hier einem ausschließlich subjektiven Gefahrenbegriff der Vorzug zu geben mit der Folge der zwingenden Rechtswidrigkeit des Einschreitens. Es mag zwar zunächst befremden, dass eine Gefahrenabwehrmaßnahme trotz eines tatsächlich abgewehrten Schadens rechtswidrig sein soll. Indes: Mit rechtsstaatlichen und grundrechtlichen Standards fundamental unvereinbar erscheint erst recht, wenn der Staat Grundrechtseingriffe mit der Gewogenheit des Zufallsgenerators legitimiert. 


\section{d) Bewertung}

Die Analyse zeigt, dass bei Gefahrenverdacht sowohl das Einschreiten als auch das Nichteinschreiten dem Risiko ${ }^{43}$ eines späteren Rechtswidrigkeitsurteils ausgesetzt ist, sofern man den modifizierten subjektiven oder den rein objektiven Gefahrenbegriff zugrundelegt. Nur ein ausschließlich subjektiver Gefahrenbegriff würde bereits ex-ante stets Klarheit über die Rechtslage gewährleisten: Ein Nichteinschreiten bei Gefahrenverdacht wäre unabhängig von den tatsächlichen Verhältnissen immer rechtmäßig (aufgrund pflichtgemäß verneinter hinreichender Wahrscheinlichkeit), ein Einschreiten immer rechtswidrig.

\section{Reaktionen des Gesetzgebers und der Lehre auf die Herausforderungen}

Jedoch widerspricht ein ausschließlich subjektiver Gefahrenbegriff nicht nur der überwiegenden Meinung. Zudem wird als rechtspolitisch unbefriedigend empfunden, wenn die Polizei trotz (rechtlich unzureichender!) Anzeichen für einen bevorstehenden Schaden »tatenlos zusehen « würde. Auf den vermeintlichen Missstand haben Gesetzgebung, Rechtsprechung und Schrifttum in vielfältiger Weise reagiert.

Der Gesetzgeber hat vermehrt Befugnistatbestände mit einem verminderten Grad der geforderten Gewissheit geschaffen. Die Rechtsprechung entnimmt bereits der Generalklausel Eingriffsbefugnisse im Falle eines Gefahrenverdachts. Große Teile des Schrifttums sind dem bislang gefolgt.

\section{a) Befugnistatbestände mit vermindertem Gewissheitsgrad}

Die Polizeigesetze kennen Befugnisnormen, deren Tatbestand den Grad der geforderten Gewissheit gegenüber einer Gefahr ausdrücklich herabsetzt: Die Figur der »Tatsachen/tatsächlichen Anhaltspunkte ${ }^{44}$, die eine bestimmte Annahme rechtfertigen«. Eingebürgert hat sich der Begriff

$43 \mathrm{Zu}$ diesem Schenke (Fn. 25), § 3 Rn. 79a.

44 Ein rechtlicher Unterschied zwischen den Formulierungen besteht nicht, s. Kral (Fn. 9), 120. A. A. Möstl/Mühl/Leggereit BeckOK PolR Hessen, 7. Ed., § 30 HSOG Rn. 8 ff. (zurückhaltend bzgl. praktischer Auswirkungen eines Unterschieds). Bäuerle ebd. § 13 HSOG Rn. $56.1 \mathrm{ff}$. vermerkt die fehlende gesetzliche Systematik bei der Verwendung der Formulierungen; ebenso Möstl JURA 2011, 840 (846). Siehe VGH Kassel, Beschl. v. 1. 2. 2017 - 8 A 2105/14.Z, juris Rn. 51. der »Vorfeldbefugnisse ${ }^{45}$ Ob die Figur einen Gefahrenverdacht markiert oder eine noch weiter vorgelagerte Eingriffsschwelle, ist umstritten (dazu noch unter bb)).

\section{aa) Historische Entwicklung}

Das Gesetz über den Bundesgrenzschutz (BGSG) von $1972^{46}$ - welches erstmalig materielle Befugnisnormen enthielt - kannte zwar bereits den Gewissheitsgrad der »Tatsachen, die eine bestimmte Annahme rechtfertigen«. Betroffen war indes nicht die geforderte Gewissheit eines Schadenseintritts, sondern die zu erwartende Effektivität des polizeilichen Eingriffs. So hieß es in $\S 20$ Abs. 2 BGSG (§ 45 Abs. 1 BPolG g.F.):

»Bei Gefahr im Verzug [...] kann der Bundesgrenzschutz eine Wohnung durchsuchen, um eine Person in Gewahrsam zu nehmen [...], wenn Tatsachen darauf schließen lassen, daß sich die Person [...] in der Wohnung befindet.«

Nachdem in den Ländern zunächst das preußische Polizeiverwaltungsgesetz fortgalt, wurden allmählich eigene Landespolizeigesetze erlassen. Tatbestände mit vermindertem Gewissheitsgrad fanden sich anfangs kaum. Das Hessische Polizeigesetz von $1954^{47}$ gestattete die Sicherstellung bei »hinreichendem Verdacht«, etwa wenn der Gegenstand zur Begehung einer mit Strafe bedrohten Handlung gebraucht oder verwertet werden soll (§ 23 Abs. 1). Das HSOG $1990^{48}$ aktualisierte die Begriffswahl und spricht von tatsächlichen Anhaltspunkten, die die Annahme rechtfertigen, dass die Sache zur Begehung einer Straftat oder Ordnungswidrigkeit gebraucht oder verwertet werden soll.

$\S 30$ Abs. 3 PolG NRW $1969^{49}$ kannte eine »jederzeitige« Betretungsbefugnis für Wohnungen zum Zwecke der Gefahrenabwehr, die »als Herbergen oder Versammlungsorte bestrafter Personen oder als Schlupfwinkel für Glücksspiel, Schmuggel, Rauschgifthandel oder gewerbsmäßige Unzucht bekannt sind «. ${ }^{50}$ Nach Auffassung des Gesetzgebers müsse indes auch für ein Betreten dieser Räumlichkeiten ein konkreter Gefahrenanlass vorliegen. Das Gesetz unterstelle nur, dass dann ohne nähere Prüfung eine »dringende« Gefahr nach Art. 13 Abs. 3 [Abs. 7 g.F.] GG

45 Zum Begriff insb. Kral (Fn. 9), $71 \mathrm{ff}$.

46 Gesetz über den Bundesgrenzschutz vom 18. 8. 1972 (BGBl. I S. 1834).

47 Hessisches Polizeigesetz vom 10. 11. 1954 (GVBl. S. 203).

48 HSOG vom 26. 6. 1990 (GVBl. S. 197, 534).

49 PolG NRW vom 28. 10. 1969, GV. NW. S. 740 (Bek. der Neufassung durch G vom 8. Juli 1969 [GV. NW. S. 521]).

50 Dazu Funk/Werkentin KJ 9 (1976), 407 (413). 
vorliegt. ${ }^{51}$ Entsprechend argumentierte der bayerische Gesetzgeber ${ }^{52}$ für die fast gleichlautende Vorschrift ${ }^{53} \mathrm{im}$ PAG 1954. Die dringende Gefahr im Sinne des Grundgesetzes umfasst nur die abstrakte Gefahr, nicht den Gefahrenverdacht (zur Unterscheidung bei bb)). In Berlin vermerkte der Gesetzgeber für § 24 Abs. 3 ASOG 1975, ${ }^{54}$ dass für die Befugnis zum Betreten derartiger Wohnungen eine konkrete Gefahr ausdrücklich nicht vorzuliegen braucht und die Eröffnung der Gefahrenabwehraufgabe ausreiche. ${ }^{55} \mathrm{In}$ diesem Gesetz erfolgt dann auch für einzelne Befugnisse die Verminderung des Gewissheitsgrades anhand der Figur der »Tatsachen, die eine bestimmte Annahme rechtfertigen " - ebenso wie im Musterentwurf für ein einheitliches Polizeigesetz 1976 (etwa für die gefährdeten/ verrufenen Orte). ${ }^{56}$

Einen Bedeutungsschub erhielt die Figur mit den informationellen Spezialbefugnissen als Reaktion auf das Volkszählungsurteil. ${ }^{57}$ Die Entwicklung hat ihren Höhepunkt gefunden in den Befugniserweiterungen zur Terrorismusbekämpfung im Bundeskriminalamtsgesetz, ${ }^{58}$ die vom Bundesverfassungsgericht teilweise beanstandet wurden. ${ }^{59}$

51 Gesetzentwurf, LT-Drs. 6/285, S. $22 \mathrm{zu} \S 16$ l Abs. 3.

52 Bay. Landtag, Tagung 1953/54, Beil. 4660 v. 6. 10. 1953, S. 27. Art. 37 Abs. 2 BayPAG 1954 verweist für die Tatbestandsvoraussetzung auf Art. 5 Abs. 2. Dieser nennt unter anderem die Straftatenverhütung (Nr. 1). Die Gesetzesbegründung legt indes nahe, dass hierunter noch nicht - wie heute - eine Eingriffsschwelle unterhalb der Gefahr (etwa Möstl/Kugelmann/Gusy/Worms PolR NRW, 5. Ed., §1 PolG Rn. 241) gemeint war. Vielmehr wurde der Begriff in ausdrücklicher Gegenüberstellung zur »Unterbindung « verwendet. Diese gelte der Störung, die Verhütung einem Einschreiten vor Beginn der Tat (S. 16f.).

$53 \S 37$ Abs. 2 BayPAG vom 16. 10. 1954 (GVBl. S. 237).

$54 \S 24$ Abs. 3 ASOG Bln vom 11. 2. 1975 (GVBl. S. 688).

55 AbgHs. Berlin, Drs. 6/1569 vom 18. 10. 1974, S. 22 (unter Bezugnahme auf die o.g. Vorschriften in NRW und Bayern).

56 Dazu Funk/Werkentin KJ 9 (1976), 407.

57 BVerfGE 65, 1.

58 Unterabschnitt zur »Abwehr von Gefahren des internationalen Terrorismus«, eingefügt durch Art. 1 Nr. 5 Gesetz zur Abwehr von Gefahren des internationalen Terrorismus durch das Bundeskriminalamt vom 25. 12. 2008 (BGBl I 2008, S. 3083). Zur Neufassung des Gesetzes siehe den Nw. in Fn. 59. Zu neuen Befugnissen Glaser JURA 209, $742 \mathrm{ff}$.

59 BVerfGE 141, 220. Der Gesetzgeber hat mit dem Gesetz zur Neustrukturierung des Bundeskriminalamtgesetzes vom 1. Juni 2017 (BGBl. I 1354) reagiert, das am 25. Mai 2018 in Kraft tritt (Art. 13 Abs. 1). $\mathrm{Zu}$ Beanstandungen führten $u$.a. legistische Unsicherheiten im Umgang mit der Reduzierung des Gewissheitsgrads, dazu Meyer JZ 2017, 429 (430 ff.).

\section{bb) Rechtsprechung und Schrifttum}

Die Eingriffsvoraussetzung der »Tatsachen, die eine Annahme rechtfertigen « wird statt als Gefahrenverdacht teilweise als abstrakte Gefahr ${ }^{60}$ oder als eine Eingriffsschwelle noch unterhalb des Gefahrenverdachts begriffen. ${ }^{61}$

Eine abstrakte Gefahr liegt vor, wenn ein Verhalten, welches im Allgemeinen nicht schadensträchtig ist, in Bezug auf eine bestimmte Situation typischerweise doch $\mathrm{zu}$ einem Schaden führt. Sie soll Gefahrenabwehr gestatten, ohne in jedem Einzelfall das tatsächliche Vorliegen dieser Schutzgutbedrohung nachweisen $\mathrm{zu}$ müssen. ${ }^{62}$ Deshalb sieht der Gesetzgeber den Erlass untergesetzlicher Geoder Verbotsnormen als Maßnahme zur Abwehr einer abstrakten Gefahr vor. Erlässt der Verordnungsgeber solche Normen, werden diese zu einem Bestandteil der Rechtsordnung und damit auch des polizeilichen Schutzguts »Öffentliche Sicherheit«. Ein Verstoß gegen die Verordnung bedeutet daher als Rechtsbruch eine konkrete Gefahr, gegen die die Polizeibehörde einschreiten kann. Die Frage, ob im betreffenden Einzelfall auch das abstrakte Schutzgut selbst konkret bedroht ist, kann die Behörde daher ignorieren.

Fall 9: Im örtlichen See wird eine biologische Belastung nachgewiesen. Eine Verordnung verbietet daher das Baden im See. ${ }^{63}$ Verstößt ein Besucher gegen das Badeverbot, kann die Behörde gegen diesen Rechtsbruch einschreiten. Darauf, ob in diesem Einzelfall auch tatsächlich eine konkrete Gesundheitsgefahr besteht, kommt es nicht an.

Vorfeldbefugnisse sind dagegen meist informationelle Befugnisse, die der Sachverhaltsaufklärung im Einzelfall dienen. Ein möglicherweise bereits in Gang befindliches Geschehen soll also gerade näher untersucht statt ignoriert werden. ${ }^{64}$ Zwar bewirken einige der Vorfeldbefugnisse eine Aufhebung der Anonymität (Identitätsfeststellung an verrufenen Orten; Videoüberwachung), deren Abschreckungswirkung geeignet ist, einen schadensträchtigen Geschehensablauf bereits zu unterbrechen (geplante Straftatbe-

60 OVG Koblenz NJW 2016, 2820 (2826, Abs. 99); OVG Hamburg NVwZ-RR 2015, 695 (702f.); VG Hannover, Urteil v. 9. 6. 2016 - 10 A 4629/11, juris Rn. 88; Kingreen/Poscher (Fn. 3), § 4 Rn. 52f., § 14 Rn. 32, 116.

61 Thiel (Fn. 24), § 8 Rn. 63 (dazu unten Fn. 69); auch Albers (Fn. 9), bes. S. 254; Kral (Fn. 9), 57; Neumann Vorsorge und Verhältnismäßigkeit, 1994, 30; Trute GS Jeand'Heur, 1999, 403 (408).

62 So BVerwGE 116, 347 (351f.). Vgl. Bäcker (Fn. 14), 211ff.; Albers (Fn. 9), $39 \mathrm{f}$.

63 Art. 27 Abs. 1 LStVG, § 46 Abs. 1 ThürOBG.

64 Entspr. Einwände gegen die Zuordnung zur abstrakten Gefahr bei Möstl/Kugelmann/Gusy/Worms (Fn. 52), § 1 Rn. 166; Möstl DVBl 2007, 581 (587). 
gehung). Beim Aufenthaltsverbot ${ }^{65}$ ist die Unterbrechung des Geschehensablaufs sogar die alleinige Wirkungsweise. Indes geschieht dies durch Einzelakt, nicht durch Rechtsverordnung. Vor allem aber unterscheidet sich der schadensträchtige Kausalnexus von dem der abstrakten Gefahr. Das von einer Verordnung verbotene Verhalten ist unmittelbar schadensträchtig (etwa das Baden im Fall 9). Dies trifft auf einen (verbotenen oder deanonymisierten) bloßen Aufenthalt gerade nicht zu. Hier bedarf es einen vom Aufenthalt unabhängigen Willensentschluss zu einer Schädigungshandlung. Der Aufenthalt ist mit der Straftatenbegehung lediglich positiv korreliert, das heißt er kann als Prognosegrundlage den Verdacht einer konkreten Gefahr begründen. ${ }^{66}$

\section{cc) Bewertung}

Nahe liegt daher, die Figur als Gefahrenverdacht zu deuten. Die Vorfeldbefugnisse gelten einem möglicherweise in Gang befindlichen schadensträchtigen Geschehen, mit anderen Worten: Es um den Verdacht einer konkreten Gefahr. ${ }^{67}$ Im Unterschied zum ungeschriebenen ${ }^{68}$ Gefahrenverdacht mag sich die potentielle Gefahrenlage raumzeitlich und inhaltlich erst in geringerem Maße abzeichnen. Der Begriffsverwendung steht dies nicht entgegen. ${ }^{69}$

$65 \S 27$ a PolG BW, § 29 Abs. 2 ASOG, § 16 Abs. 2 BbgPolG, § 14 Abs. 2 BremPolG, § 12 b Abs. 2 SOG (Hambg.), § 31 Abs. 3 HSOG, § 52 Abs. 3 SOG M-V, § 17 Abs. 4 Nds. SOG, § 34 Abs. 2 PolG NRW, § 13 Abs. 3 POG (Rh.-Pf.), § 12 Abs. 3 SPolG (Saarl.), § 21 Abs. 2 SächsPolG, § 36 Abs. 2 SOG LSA, § 201 Abs. 2 LVwG (S.-H.), § 18 Abs. 3 PAG (Thür.). Siehe zu dieser Befugnis VGH Kassel, Beschl. v. 1. 2. 2017 - 8 A 2105/14.Z.

66 Ausf. Meyer JZ 2017, 429 (432f.). Ähnl. zum Kausalnexus Bonin (Fn. 9), 216f.

67 In diesem Sinne Möstl/Kugelmann/Gusy/Worms (Fn. 52), §1 Rn. 166 (»typisierte Sonderform des Gefahrverdachts«); Möstl DVBl 2007, 581 (587 f.); ders. DVBl 2010, 808 (810 f.); ders. (Fn. 8), 67 f., 215 (»Vorfeld eines konkreten Gefahrenverdachts«); Schoch/ders. (Fn. 5), Rn. 147; Kugelmann Polizei- und Ordnungsrecht, 2. Aufl. 2011, 5. Kap. Rn. 99; Geis/Götz (Fn. 3), § 6 Rn. 38. Ausf. Meyer JZ 2017, 429 (433). Krit. Bäcker (Fn. 14), 126.

$68 \mathrm{Zu}$ diesem unter $\mathrm{b})$.

69 A.A. Thiel (Fn. 24), § 8 Rn. 63. Eine Einordnung der »Tatsachen, die eine Annahme rechtfertigen« als Gefahrenverdacht impliziere einen »Gefahrenbezug der Norm«, der den Vorfeldbefugnissen aber gerade fehle. Diese Annahme ist indes nicht zwingend. „Gefahrenverdacht « als Bezeichnung eines bestimmten Gewissheitsgrades kann durchaus eine dogmatisch eigenständige Eingriffsschwelle markieren. Der Begriff »Gefahrenverdacht « enthält lediglich deshalb das Wort "Gefahr«, weil die Definition des Gefahrenverdachts die Gefahrendefinition als ihren Ausgangspunkt verwendet, welcher dann aber modifiziert wird: Aus dem »Vorliegen einer hinreichenden Wahrscheinlichkeit...» wird die »Möglichkeit des Vorliegens einer hinreichenden Wahrscheinlichkeit...».
Bereits der Gefahrenbegriff verarbeitet mit der Je-destoFormel unterschiedliche Gewissheitsgrade.

\section{b) Gewöhnlicher Gefahrentatbestand und Gefahrenverdacht}

In der Rechtsprechung und in weiten Teilen des Schrifttums werden Eingriffe bei Gefahrenverdacht auch dann für zulässig gehalten, wenn keine Vorfeldbefugnis einschlägig ist. Vielmehr sei eine geeignete Befugnis schon in der Generalklausel bzw. in den Spezialbefugnissen als ungeschriebener Annex des Gefahrentatbestands enthalten. Der Verhältnismäßigkeitsgrundsatz soll eine Beschränkung auf Gefahrenerforschungseingriffe gebieten. ${ }^{70}$ Bei hohem Stellenwert der bedrohten Rechtsgüter sollen indes endgültige Gefahrenabwehrmaßnahmen zu treffen sein. ${ }^{71}$ Dieses Verständnis war zu Recht schon immer deutlicher Kritik ausgesetzt gewesen, die zuletzt wieder lauter wird. ${ }^{72}$

Der Gesetzgeber bestätigt mit dem wahrscheinlichkeitsbasierten Gefahrentatbestand, dass er sich über die Unsicherheit zukünftiger Entwicklungen im klaren ist. ${ }^{73}$ Deshalb zieht dieser Tatbestand eine gewollte Grenze zwischen dem hinnehmbaren und dem nicht mehr hinnehmbaren Maß an Ungewissheit. Die weitere Ausdehnung des hinnehmbaren Maßes entspricht somit nicht Sinn und Zweck der Regelung. Eine methodische Rechtfertigung der Übersteigung der Wortlautgrenze ist hier also nicht in Sicht und erfolgt diese contra legem. ${ }^{74}$ Zudem ermächtigen die Befugnisnormen zu Eingriffen in Grundrechte. ${ }^{75}$ Auch dies spricht gegen eine erweiternde Auslegung.

70 BVerwGE 116, 347 (351ff.); VGH München BayVBl 2017, 303 (303, Abs. 17); VGH Kassel, Beschl. v. 1. 2. 2017 - 8 A 2105/14.Z, juris Rn. 54; OVG Bautzen, SächsVBl 2014, 240 (241, Abs. 6); VGH Mannheim VBlBW 2014, 56 (57); OVG Koblenz, Urt. v. 24. 1. 2013 - 7 A 10816/12, juris Rn. 30; OVG Lüneburg OVGE MüLü 55, 456 (461) = NdsVBl 2013, 68 (69); OVG Saarlouis, Beschl. v. 31. 3.2004 - 2 N 2/03, juris Rn. 88 ff.; VG Berlin, Urt. v. 25. 8. 2016 - 1 K 318.14, juris Rn. 19; VG Köln, Urt. v. 10. 12. 2015 - 20 K 7847/13, juris Rn. 71 ff. Offengelassen in OVG Hamburg NVwZ-RR 2009, 878 (882). Geis/Götz (Fn. 3), § 6 Rn. 31; Schoch/ders. (Fn. 5), Rn. 147; Bäcker (Fn. 14), 97 ff.

71 VGH Mannheim VBlBW 2014, 56 (57); Lisken/Denninger/Rachor/ Denninger Hdb Polizeirecht, 5. Aufl. 2012, D Rn. 48.

72 Möstl JURA 2005, 48 (53 in Fußn. 43); ders. (Fn. 8), 173, 181, 184; Di Fabio Risikoentscheidungen im Rechtsstaat, 1994, 316ff.; Jochum/ Fritzemeyer/Kau/Ibler FS Hailbronner, 2013, 737 ff.; Schenke (Fn. 25), $\S 3$ Rn. 88 ff.; Thiel (Fn. 24), § 8 Rn. 61 ff.; Wapler DVBl 2012, $86 \mathrm{ff}$. 73 Ebenso Schenke (Fn. 25), § 3 Rn. 89; Thiel (Fn. 24), § 8 Rn. 64. 74 A. A. Schoch/ders. (Fn. 5), Rn. 147.

75 Ebenso Paeffgen GA 2014, 638 (644); Schenke (Fn. 25), § 3 Rn. 88 a. 
Hält der Gesetzgeber diese Rechtslage für sicherheitspolitisch unbefriedigend, ist er nicht gehindert, Eingriffstatbestände zu erweitern. ${ }^{76}$ Der Gefahrentatbestand taugt nicht als Eingriffsgrundlage bei Gefahrenverdacht.

\section{Fazit}

Der herrschende subjektive Gefahrenbegriff ermöglicht Polizeibeamten, die eingreifende Maßnahmen beabsichtigen, das gesetzliche Tatbestandserfordernis einer Gefahr gezielt zu beachten. Der Begriff verhindert also, dass die Rechtmäßigkeit staatlichen Handelns bloßer Zufall bleibt, und er verdient wegen dieser rationalisierenden Funktion Zustimmung.

Fälle des Gefahrenverdachts sind gewöhnlichen Gefahrentatbeständen nicht subsumierbar. Entgegen stehen die Wortlautgrenze und der Sinn und Zweck des Gefahrentatbestands. Die Schaffung polizeilicher Eingriffsbefugnisse bei Gefahrenverdacht ist dem Gesetzgeber vorbehalten. Jüngere polizeigesetzliche Spezialbefugnisse stellen bereits auf einen Gefahrenverdacht als Eingriffsschwelle ab.

76 Jochum/Fritzemeyer/Kau/Ibler (Fn. 72), 743 ff.; Wapler DVBl 2012, 86 (88). 